\title{
Computing Efficiency Improvement in Monte Carlo Simulation of a 12 MV Photon Beam Medical LINAC
}

\author{
M. Zoubair ${ }^{*}$, T. El Bardouni ${ }^{1}$, O. Allaoui ${ }^{1}$, Y. Boulaich ${ }^{1}$, B. El Bakkari ${ }^{1}$, \\ C. El Younoussi ${ }^{1}$, H. Boukhal ${ }^{1}$, E. Chakir ${ }^{2}$ \\ ${ }^{1}$ ERSN-LMR, Faculty of Sciences, University Abdelmalek Essaadi, Tetouan, Morocco \\ ${ }^{2}$ Faculty of Sciences, University Ibn Tofail, Kénitra, Morocco \\ Email: "zoubair-mariam@hotmail.fr
}

Received October 11, 2012; revised November 18, 2012; accepted December 4, 2012

\begin{abstract}
Variance reduction techniques (VRTs) have been tremendously successful when applied to Monte Carlo radiation transport codes for which the computation time constitutes an important and a problematic parameter. In fact, many Monte Carlo calculations absolutely require variance reduction methods to achieve practical computation times. The MCNPX code has a fairly rich set of variance reduction techniques; the most known are transport cutoffs, interaction forcing, Bremsstrahlung splitting and Russian roulette. Also, the use of a phase space seems to be appropriate to reduce enormously the computing time. This work deals with the use of VRTs provided by MCNPX code for the simulation of a clinical linear electron accelerator (LINAC). Differences between various sets of VRTs are investigated. Combination between VRTs and PS is also analyzed during this study. Analysis showed that the use of VRTs and PS improve the simulation efficiency by a factor greater than 700. Finally, experimental curves of depth-dose and dose profile performed in a homogeneous water phantom are compared to dose distributions computed by use of MCNPX Monte Carlo code.
\end{abstract}

Keywords: Variance Reduction Techniques; Phase Space; LINAC; Monte Carlo Simulation

\section{Introduction}

Monte Carlo (MC) methods are considered to provide the best calculation engine available today in medical radiation physics [1,2].

The Monte Carlo method is, by its nature, very time consuming which constitutes the main disadvantage to their use in radiotherapy. The computation time depends on the number of particles generated, their energy, their type and the medium in which they are transported. Normally, to obtain good statistics it is required to track hundreds or thousands of millions of particles. The development of advanced computers with special capabilities for parallel calculations has opened new issues for Monte Carlo researchers. Parallel computers are becoming increasingly accessible to medical physicists. However, for many medical applications this is not enough, because CPU time is still large $[3,4]$.

The variance reduction techniques play an important role in reducing uncertainties and improving the statistical results. Note that precision is not the only require-

\footnotetext{
"Corresponding author.
}

ment for a good MC calculation but computing time is also of great importance [5].

Physists also must take care to obtain a reliable physical response in short time. The idea behind these techniques is to change the physical reality during the simulation so that more effective interactions would take place. For example, if we increase the cross-section interaction, we will get a higher number of interactions for the same number of histories. However, in some cases these techniques modify the physics of the problem and the quantity to be scored, even if it is statistically precise needs to be corrected to account for the change in the probability distributions used to describe the physics of the problem. The LANL Monte Carlo code MCNPX (Version 2.5) [6] offers several variance reduction techniques. In this paper, we applied these techniques in a linear accelerator (LINAC) simulation in order to determine the computing time gain, reduce uncertainties and improve the statistical results. First, we have compared the phase space (PS) two steps simulation to the direct head accelerator one in order to determine the PS gain and then we combined VRTs and PS for final simulation 
time enhancement. All our calculations have been done using MCNPX code.

In order to validate the results of our MC simulation, the depth doses and profile curves for a homogeneous water phantom of $30 \times 30 \times 30 \mathrm{~cm}^{3}$ were compared to experimental ones obtained at the French National Metrological Laboratory for ionizing radiation (LNHB) [7].

\section{Materials and Methods}

\subsection{Monte Carlo Methods}

Monte Carlo (MC) methods have been used in various branches of radiation therapy, from simulation of radiation therapy equipments and sources to dose calculation in various geometries. These methods which are stochastic techniques are based on the use of random numbers and probability statistics to investigate problems. Thus MC methods are a collection of different methods that perform the same process: this process involves performing many simulations using random numbers and probability distributions to get an approximation of the answer to the problem. One of the most important usages of MC methods concerns the evaluation of difficult integrals. This is especially true for multi-dimensional integrals for which few analytical computation methods are suitable to get their appropriate approximation due to their complexity. It is in these situations that $\mathrm{MC}$ approximations become a valuable tool to use; they may be able to give a reasonable approximation with higher accuracy comparatively to other formal techniques [8].

At first, the development of dedicated coupled photon electron transport codes for each specific problem required a lot of effort. Today, this is no longer necessary due to the availability of general purpose codes like ETRAN, ITS, MCNP, EGS, GEANT and PENELOPE. Most Monte Carlo systems dedicated to radiotherapy are completely or partially based on these codes. In this paper, we have an interest in the MCNPX (Monte Carlo N-Particle) code, which is developed by Los Alamos National Laboratory (LANL). MCNPX can be used to simulate many types of particles [6,9]. The photon and electron physics in the present extended version of MCNP code is identical to that in MCNP5.

\subsection{The Linac Accelerator}

In this study, we simulated a linac head dedicated to generate $12 \mathrm{MV}$ photon beam. The components of the linear accelerator head are shown in Figure 1. These components include $\mathrm{W}$ target, primary collimator, flattening filter, and secondary collimator jaws. In Figure 2, we show our generated $3 \mathrm{D}$ view of the modeled linac head associated to a water phantom with dimension of $30 \times 30 \times 30 \mathrm{~cm}^{3}$ that was placed at a source to surface

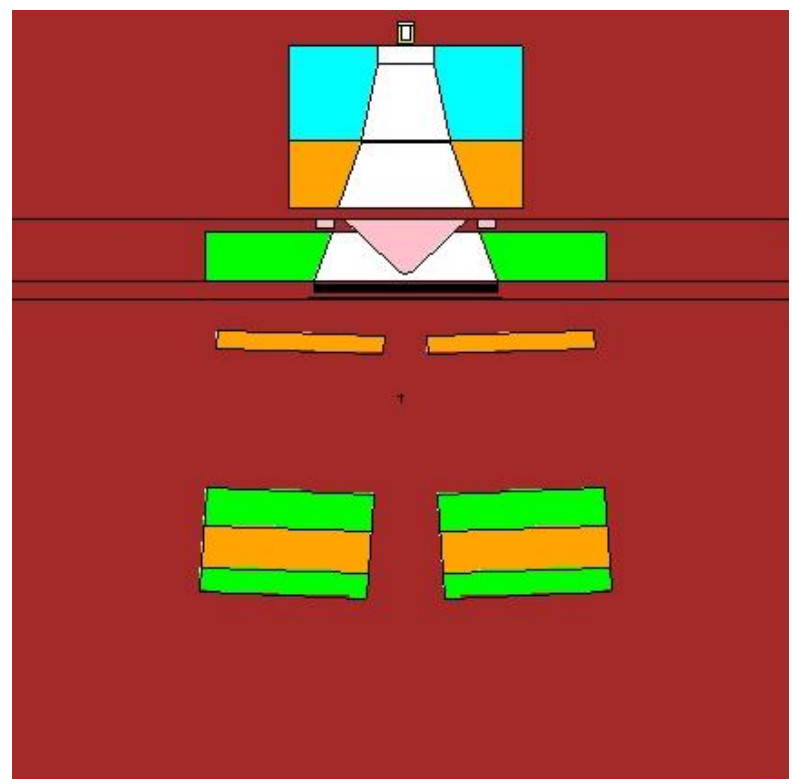

Figure 1. The schematic representation of Linac head geometry as used in our simulation of $12 \mathrm{MV}$ photon beam.

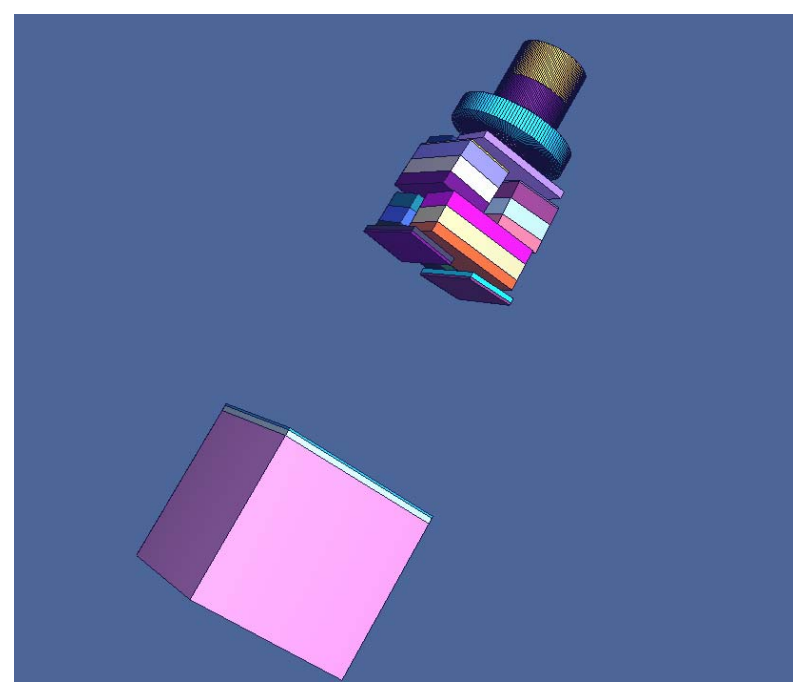

Figure 2. 3D view of our model of the Linac head and a water phantom $30 \times 30 \times 30 \mathrm{~cm}^{3}$.

distance $(\mathrm{SSD})$ of $90 \mathrm{~cm}$. The upper and lower jaws were set to create a field size of $10 \times 10 \mathrm{~cm}^{2}$ on the phantom surface.

\subsection{Computing Efficiency of a MC Calculation}

The efficiency $(\varepsilon)$ of a Monte Carlo simulation may be quantified using the Figure of Merit (FOM), which assists the user in assessing the statistical behavior of the obtained answer. The MCNPX code developers defined FOM according to Equation (1) $[4,10,11]$ :

$$
\varepsilon=\mathrm{FOM}=\frac{1}{T \cdot \sigma^{2}}
$$


where: $T$ : is the total calculation time (CPU) for $N$ histories. $\sigma$ : is the tally relative statistical uncertainty.

For a fixed computing time, the smaller is the variance the larger will be the FOM. It should be noted that $\sigma^{2}$ is proportional to $1 / N$, where $N$ is the total number of histories and $T$ is proportional to $N$. Thus, for a well converged simulation, the FOM becomes constant.

For instance, the use of variance reduction techniques and some special approximations in electron transport codes is required to obtain sufficient accuracy within acceptable calculation times [12].

\subsection{Overview of Variance Reduction Techniques}

The Variance reduction techniques are important elements of any Monte Carlo code and may be different for various applications and geometries. These techniques reduce the time of particle simulations and improve the speed of the code. There are four main categories of variance reduction techniques, detailed in the following paragraph:

Truncation Methods: Are the simplest variance reduction methods. They speed up calculations by truncating parts of space that do not contribute significantly to the solution. The simplest example concerns geometry truncation in which unimportant parts of the geometry are simply not modeled [13]. The specific truncation methods available in MCNPX are the energy cutoff, weight cutoff and time cutoff. In this work, we are interested in energy cutoff (CUT card).

Population Control Methods: These methods artificially increase the number of particles in spatial or energy regions that are important or decrease this number in the case it doesn't contribute to the score tally. In MCNPX, the specific methods of population control that we used are Geometry splitting and Russian roulette (IMP card).

Modified Sampling Methods: These methods artificially increase the likelihood of events increasing the particle of interest probability to reach the tally region. Implicit capture (PHYS card) and Bremsstrahlung biasing (BBREM card) are included in MCNPX.

Partially-Deterministic Methods: Are the most complicated class of variance reduction methods. They circumvent the normal random walk process by using deterministic-like techniques, such as next event estimators, or by controlling the random number sequence [14].

\subsection{The Phase Space}

The Monte Carlo code MCNPX is currently used to create and evaluate the PS distributions from linear accelerator head simulations; it's a method to reduce computational time in a subsequent simulation of particle transport.
The phase space, typically containing information such as energy, charge, position, direction, previous history, etc on millions of particles (photons, electrons, positrons), can be sampled for further particle transport in the rest of the geometry (linac or phantom). When the PSis situated at the bottom of the linac, it replaces effectively the linac and becomes the virtual linac. In this study, we have compared the PS two steps simulation to the direct head accelerator one in order to determine the PS gain using *F8 energy deposition tally of MCNPX code. With this tally, we can calculate the absorbed dose (MeV/g/particle) in material due to photons and electrons. In this paper, we will validate this computing time reduction technique by comparing the obtained percent depth dose (PDD), in a water phantom for a field $10 \times 10 \mathrm{~cm}^{2}$ and a $12 \mathrm{MV}$ photon beam, in both accelerated and direct calculation.

\section{Results and Discussion}

\subsection{PS Effect}

Following the methodology described in the previous section, we run two kinds of simulations: 1) direct simulation without PS and 2) accelerated simulation using the phase space. For the results quoted below, direct simulation and accelerated one were done by following $15 \times$ $10^{8}$ histories. Calculations have been run in parallel on a low cost cluster composed of 11 Core2Duo PCs using the MPI parallel protocol with up to 22 processes.

Table 1 shows a comparison between the absorbed dose at $10 \mathrm{~cm}$ within water phantom due to $12 \mathrm{MV}$ photon beam for both types of simulations. CPU time T, Relative statistical uncertainty $S(1 \sigma)$ on the absorbed dose and efficiency $\varepsilon$ are presented.

As we can see the agreement between direct and accelerated calculations is remarkable and these points out the feasibility and validity of the proposed methodology. The advantage of the PS simulation is evident in Table 1. It's shown that a similar level of uncertainty is obtained in a remarkably short time. Consequently the efficiency of calculation is improved by a factor greater than 40 .

Hence the PS allows saving computing time without any approximation made for each individual treated particle. This enhances the accuracy of calculation without altering the physical results.

We show in Figure 3 the mesh tally; it's a method in

Table 1. Comparison between results of the $12 \mathrm{MV}$ photon beam simulation with and without PS.

\begin{tabular}{ccccc}
\hline Simulation type & $T(\mathrm{~min})$ & $\begin{array}{c}\text { Absorbed dose at } 10 \mathrm{~cm} \\
\text { (MeV/g/incident } \\
\text { particle) }\end{array}$ & $S(\%)$ & $\varepsilon$ \\
\hline Without PS & 58226 & $2.26 \times 10^{-6}$ & 4.26 & 0.009 \\
With PS & 1241 & $2.33 \times 10^{-6}$ & 4.75 & 0.357 \\
\hline
\end{tabular}




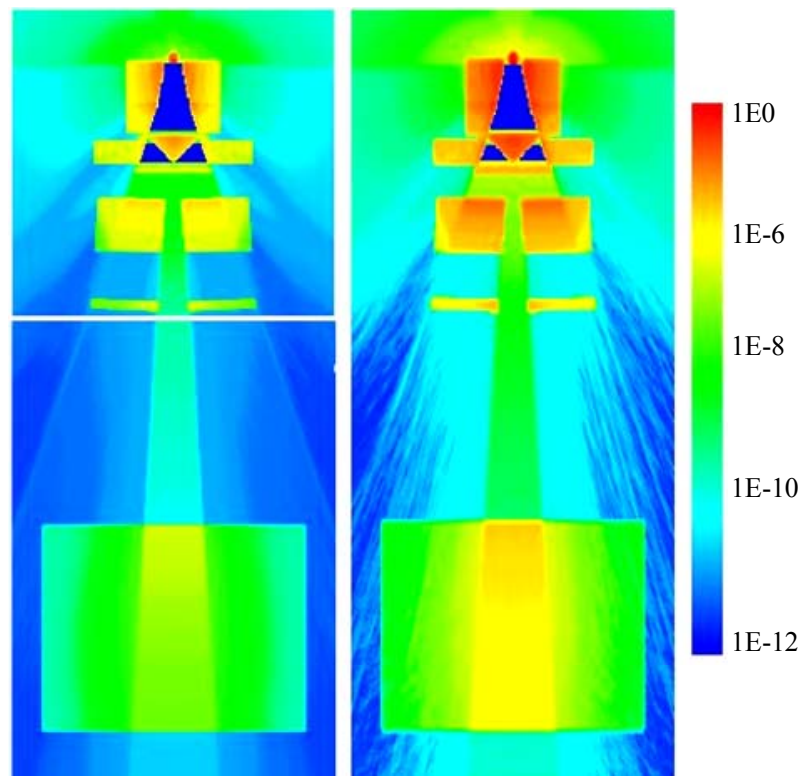

Figure 3. Relative absorbed dose from mesh tally for phase space (left) and direct (right) simulations.

MCNPX which displays graphically particle flux, dose or other quantities distribution on a rectangular, cylindrical or spherical grid overlaid on top of the standard problem geometry. To obtain this figure, we have used the first mesh type tally which scores track averaged data to estimate the absorbed dose.

We run two cases of mesh tally simulation for energy deposition per unit volume $\left(\mathrm{MeV} / \mathrm{cm}^{3} /\right.$ source particle). In Figure 3 (right part) we give the relative results of direct calculation. Whereas left part of Figure 3 provides the results of simulation that involves the PS technique for roughly the same CPU calculation time. In this case the two parts simulated separately are shown: the Linac head region above the PS level (left top) and the region below the PS (left bottom) which contains the phantom. By comparing the resolution (smoothness) of the two images we can notice the speedup of the convergence of the simulation due to PS technique.

\subsection{Variance Reduction Techniques Comparison}

In the second section of this work, we adopt the method of PS and investigate the effect of different variance reduction techniques on the calculated absorbed dose within a water phantom.

\subsubsection{Bremsstrahlung Biasing}

Bremsstrahlung interaction of electron beam on a heavy target produces many low-energy photons; however the higher energy photons are often of more interest. The use of BBREM card in MCNPX allows the generation of more high-energy photon tracks by biasing each sampling of Bremsstrahlung event toward a larger fraction of available electron energy. In this part we compare the absorbed dose calculations with and without use of the BBREM card. The effect of this card is summarized in Table 2 where we show the histories number NPS, the computing time $\mathrm{T}$ and the relative statistical uncertainty $\mathrm{S}(1 \sigma)$ on absorbed dose in the build-up region cell.

In Table 2, we observe that the relative statistical uncertainty obtained in calculation without BBREM card is higher than the one obtained in the case where Bremsstrahlung is biased. We also notice that no improvement is achieved even if we increase the histories number 4 times. However, the use of BBREM card decreases the relative statistical uncertainty by a factor of about 12 .

\subsubsection{Electron and Photon Energy Cut-Off}

Simulation of electron tracks requires large computation time. To reduce it, MCNPX code allows the use of CUT card; this card is used to specify a minimum energy, time or particle weight below which the particle is killed (X-5 Monte Carlo Team MCNP, 2003). In this work, we are interested in the energy cut-off for electrons and photons.

The previous calculations were performed with an energy cut-off of $60 \mathrm{keV}$ adopted in the transport of both photons and electrons. Looking for improvement of computation time, the energy cut-off was increased to $400 \mathrm{keV}$ for electrons and $120 \mathrm{keV}$ for photons. In Table 3 we compare the CPU times and the results obtained versus cut-off energy.

Our calculations lead to a good agreement between the values of absorbed dose in build-up cell for both CUT cases as shown in Table 3. Increasing the cut-off energy allows to reach the same statistical uncertainty with a gain of $52 \%$ in calculation time.

\subsubsection{Physics Card}

The PHYS card in MCNPX is used to specify energy

Table 2. Comparison of simulations with and without BBREM card for the $12 \mathrm{MV}$ photon beam.

\begin{tabular}{cccc}
\hline Simulation & NPS & $T(\mathrm{~min})$ & $S(\%)$ \\
\hline \multirow{2}{*}{ Without BBREM } & $15 \times 10^{8}$ & 775.7 & 0.50 \\
& $60 \times 10^{8}$ & 3116.1 & 0.44 \\
With BBREM & $15 \times 10^{8}$ & 3410.7 & 0.04 \\
\hline
\end{tabular}

Table 3. CPU times comparison for different energy cut-off values in the simulation of $12 \mathrm{MV}$ photon beam.

\begin{tabular}{ccc}
\hline Energy cutoff & $T(\mathrm{~min})$ & $\begin{array}{c}\text { Absorbed dose in the build-up cell } \\
(\mathrm{MeV} / \mathrm{g} / \text { incident particle) }\end{array}$ \\
\hline $\begin{array}{l}\text { CUT: E } 0.06 \mathrm{MeV} \\
\text { CUT: P } 0.06 \mathrm{MeV}\end{array}$ & 2634.4 & $2.43 \times 10^{-6}( \pm 0.5 \%)$ \\
CUT: E $0.4 \mathrm{MeV}$ & 1260.7 & $2.44 \times 10^{-6}( \pm 0.5 \%)$ \\
CUT: P $0.12 \mathrm{MeV}$ & & \\
\hline
\end{tabular}


limits for physics approximation and other parameters to be used in the physics treatment of particles transport. To each kind of particles corresponds different parameters sets which are specified as inputs for PHYS card. In this study, we focus our interest on controlling the physics of electrons and we studied the main two parameters of PHYS card which are BNUM for controlling the Bremsstrahlung photons production and RNOK to control the production of knock-on (delta rays) electrons.

\subsubsection{BNUM Parameter}

In MCNPX, the Bremsstrahlung photon production is controlled by the parameter BNUM of PHYS card and is used to bias the sampling of Bremsstrahlung photons produced along the electron substeps. The default value BNUM $=1$ results in the analogue sampling of Bremsstrahlung tracks. If BNUM $>1$, the number of Bremsstrahlung photons produced is BNUM times the number that would be produced in the analogue case. If the number of tracks is increased, an appropriate weight reduction is made. If BNUM $=0$, the production of Bremsstrahlung photons is turned off. To find the optimum value of BNUM for PS file generation, we recorded the number of tracks on the scoring surface for several runs, corresponding to different values of BNUM and the same number of simulated primary electrons. We compared the scores for increasing BNUM values. The number of tracks increases with BNUM as shown in Figure $\mathbf{4}$ and after a given value it reaches its asymptotic behavior.

In Table 4, we show the values of absorbed dose at 10 $\mathrm{cm}$ in a water phantom for three different values of BNUM. It's noticed that significant improvement in the statistical uncertainty s can be obtained for larger values of BNUM parameter. Hence, large values of BNUM will increase the computation time. As a compromise between

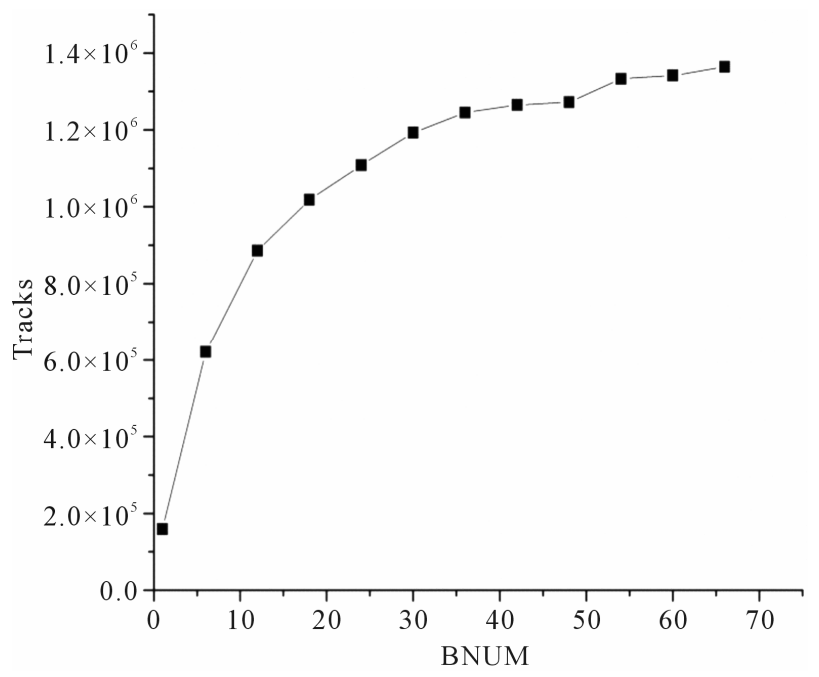

Figure 4. Number of tracks variation versus BNUM parameter for $12 \mathrm{MV}$ photon beam.
CPU time and statistical uncertainty level a BNUM value of 60 was selected for further calculations.

\subsubsection{RNOK Parameter}

Knock-on electrons production can be controlled in MCNPX by the RNOK parameter. The default value $\mathrm{RNOK}=1$ produces an analogue sampling of knock-on electrons. If $\mathrm{RNOK}=0$, the production of Knock-on electrons is turned off. An entry $>1$ for RNOK parameter leads to the production of RNOK times the analogue number of knock-on electrons. We studied the effect of these electrons, during PS computation, on absorbed dose in build-up cell region. The results are summarized in Table 5.

Turning on Knock-on electrons production during the calculation of the phase space, leads to PDD values in build-up region more accurate than values obtained in the case these electrons generating is ignored. The results show that knock-on electrons of low energy have an effect which is not negligible on PDD convergence at the entrance of the phantom.

\subsection{Combination of Variance Reduction Techniques and Phase Space}

In this part of this work we determine the gain of CPU time by combining both PS method and different techniques of variance reduction studied before; so we have recalculated the PDD with all the discussed techniques (accelerated simulation) and compared the results to those of corresponding analogue calculation. Figure 3 presents the obtained results.

On Figure 5 we show the comparison between the depth dose distributions obtained for the $12 \mathrm{MV}$ photon beam in both types of simulations. Therein, red circle symbols represent the results calculated using the PS with variance reduction techniques, whereas blue squares

Table 4. Comparison of the absorbed dose at $10 \mathrm{~cm}$ for different values of BNUM for the $12 \mathrm{MV}$ photon beam.

\begin{tabular}{ccc}
\hline BNUM & $\begin{array}{c}\text { Absorbed dose at } 10 \mathrm{~cm} \\
(\mathrm{MeV} / \mathrm{g} / \text { incident particle })\end{array}$ & $S(\%)$ \\
\hline 30 & $2.26 \times 10^{-6}$ & 0.58 \\
45 & $2.23 \times 10^{-6}$ & 0.47 \\
60 & $2.24 \times 10^{-6}$ & 0.41 \\
\hline
\end{tabular}

Table 5. The effect of knock-on electrons on absorbed dose for the $12 \mathrm{MV}$ photon beam.

\begin{tabular}{ccc}
\hline RNOK & $\begin{array}{c}\text { Absorbed dose in build-up cell } \\
(\mathrm{MeV} / \mathrm{g} / \text { incident particle })\end{array}$ & $S(\%)$ \\
\hline RNOK $=1$ & $2.44 \times 10^{-6}$ & 0.56 \\
RNOK $=0$ & $2.56 \times 10^{-6}$ & 2.44 \\
\hline
\end{tabular}




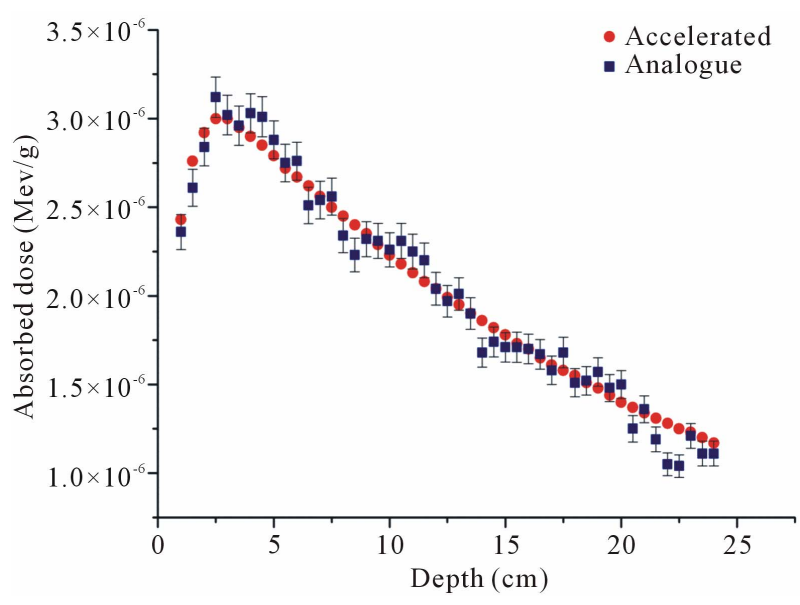

Figure 5. Depth dose in the beam axis as a function of the depth in the water phantom for the $12 \mathrm{MV}$ photon beam. Red circle symbols represent the results calculated using the phase space with variance reduction techniques. Blue squares represent the results of an analogue simulation. The error bars in the case of the accelerated simulation are small compared to the analogous simulation.

represent the results of an analogue simulation where neither variance reduction nor PS techniques were used. The PDD curve is remarkably smoother in the accelerated case than the analogue one and the uncertainties on the obtained results within the accelerated scheme are very low when compared to those quoted for the analogue simulation.

We summarized the effect of space phase and variance reduction techniques in Table 6, where we show CPU time $T$, relative statistical uncertainty $S(1 \sigma)$ for absorbed dose at $10 \mathrm{~cm}$ and simulation efficiency $\varepsilon$. This table summarizes the study carried in this paper. We found a very important gain of CPU time when we enhance our simulation scheme to include the PS and variance reduction techniques. The efficiency is increased by a factor greater than 700 .

As summary, Figure 6 represents PDD results in the accelerated simulation which is performed in two steps. We combined the PS and variance reduction techniques to calculate the PDD within a water phantom. The curve is very smooth and the statistical uncertainties ( $1 \sigma$ standard deviation) are smaller than the symbol size for the calculated results, which has an uncertainty of only $0.5 \%$.

\subsection{Modeling Validation}

\subsubsection{The Depth Dose and Dose Profile within a Water Phantom}

The previous conclusions were deduced from pure numerical analyses. In order to validate our computing proposal and in the aim to verify that the adopted variance reduction techniques don't alter the physics of the problem we compare Monte Carlo calculation values to

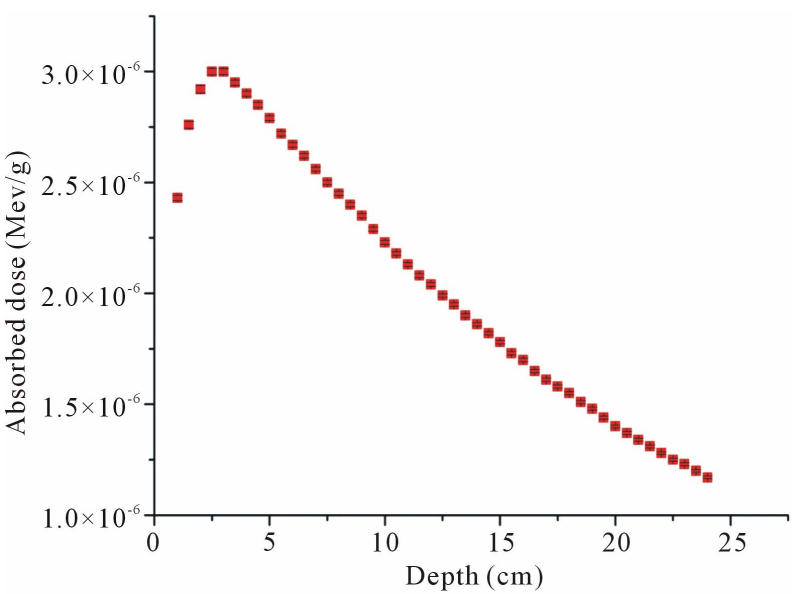

Figure 6. Depth dose in the beam axis as a function of the depth in a water phantom for the $12 \mathrm{MV}$ photon beam.

Table 6. CPU time $T$, uncertainty $S$ on absorbed dose at 10 $\mathrm{cm}$ and calculation efficiency $\varepsilon$ for the $12 \mathrm{MV}$ photon beam.

\begin{tabular}{lcccc}
\hline $\begin{array}{c}\text { Simulation } \\
\text { type }\end{array}$ & $T(\mathrm{~min})$ & $\begin{array}{c}\text { Absorbed dose at } 10 \mathrm{~cm} \\
(\mathrm{MeV} / \mathrm{g} / \text { incident } \\
\text { particle) }\end{array}$ & $S(\%)$ & $\varepsilon$ \\
\hline Analogue & 58226.4 & $2.28 \times 10^{-6}$ & 4.3 & 0.009 \\
Accelerated & 9419.2 & $2.23 \times 10^{-6}$ & 0.4 & 6.635 \\
\hline
\end{tabular}

experimental dose distributions. In the Monte Carlo calculation, we used the PS method and variance reduction techniques (BBREM, CUT, PHYS), Based on the tow parameters study of carte PHYS we decided to fix the value of $B N U M=60$ and $R N O C=1$ in the PS file and $\mathrm{BNUM}, \mathrm{RNOC}=1$ in the phantom.

In Figures 7 and 8, we show the calculated depth dose and dose profile curves compared to the experimental ones obtained for $12 \mathrm{MV}$ photon beam in water phantom in the case of a square field size of $10 \times 10 \mathrm{~cm}^{2}$. The depth dose curve is normalized to the dose at $10 \mathrm{~cm}$. The statistical uncertainties $(1 \sigma)$ associated with the simulated depth dose curve are less than $0.4 \%$ for the high dose region and less than $1 \%$ in the buildup region. The dose profile is normalized to the dose on the central axis of the beam. The statistical uncertainties $(1 \sigma)$ of the simulated profile are less than $0.4 \%$ in the central region and less than $2 \%$ in the penumbra regions.

As it can be verified in these figures, the very good agreement obtained in the comparison between the experimental values and the simulated ones, confirms the goodness of our computational simulation. This demonstrates also the efficiency of the method adopted in reducing CPU time without changing the reality of the problem.

\subsubsection{Photon Energy Spectra}

As a very good agreement has been obtained between 


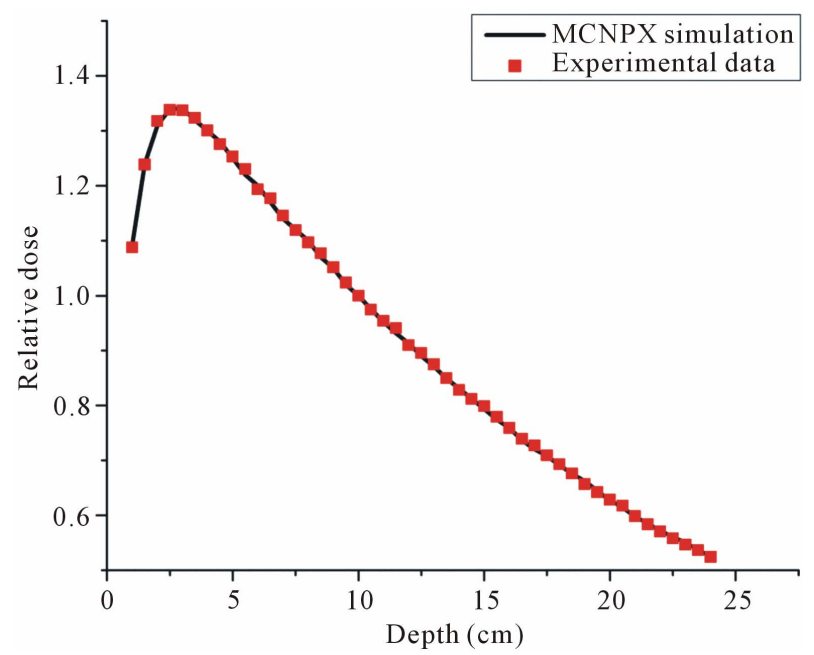

Figure 7. Comparison of calculated and experimental relative depth dose due to $12 \mathrm{MV}$ photon beam in homogeneous water phantom, for a $10 \times 10 \mathrm{~cm}^{2}$ field size. Results are normalized to the dose at the depth of $10 \mathrm{~cm}$.

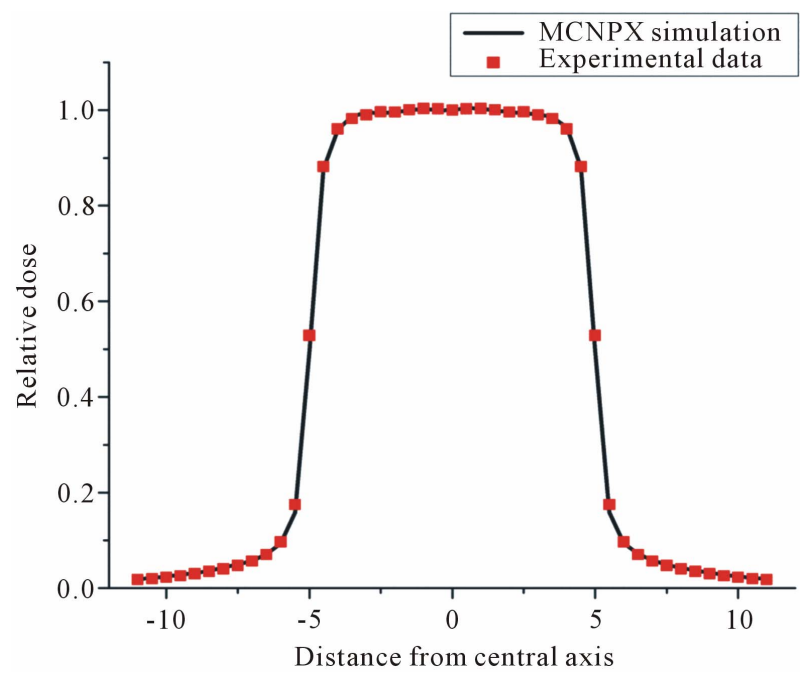

Figure 8. Comparison of calculated and measured dose profile at the depth of $10 \mathrm{~cm}$ due to $12 \mathrm{MV}$ photon beam in homogeneous water phantom, for a $10 \times 10 \mathrm{~cm}^{2}$ field size.

calculation and measurement for depth dose and dose profile curves, we propose a computed photon spectrum that could be simulated by use of MCNPX code and our model of linac head. Figure 9 shows the results obtained for energy dependent flux of a $12 \mathrm{MV}$ photon beam at 90 $\mathrm{cm}$ SSD. The whole population of the photons in spectrum was normalised to one incident electron. The Energy bins have an homogenous width of $0.120 \mathrm{MeV}$. The relative uncertainties in this case are less than $3 \%$ for almost all the energy range except few higher energy bins where photons population is poor. The weighted mean energy of photon spectrum is $3.29 \mathrm{MeV}$ which is comparable to the value $3.24 \mathrm{MeV}$ published by Blazy et al. [7] for a similar linac.

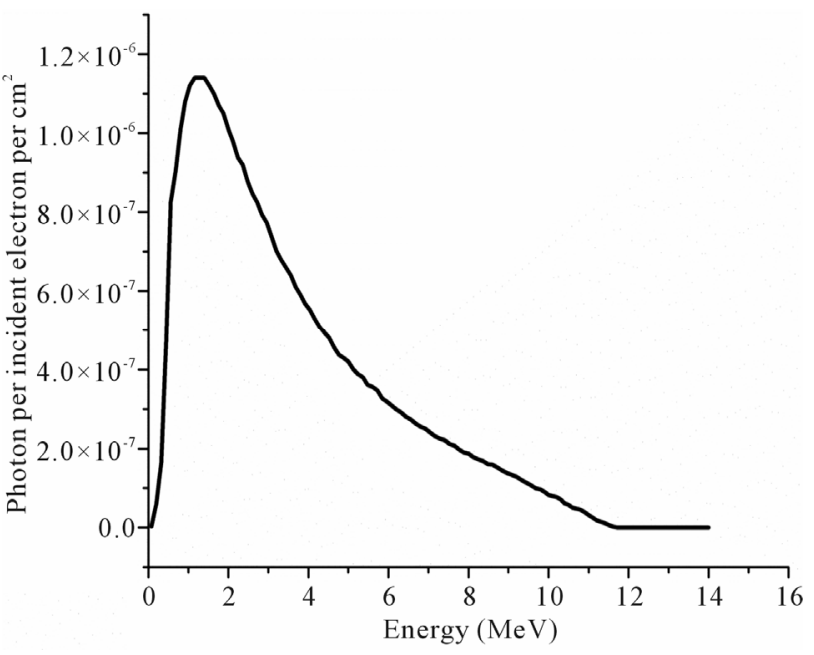

Figure 9. Monte Carlo calculated photon energy spectrum at SSD $90 \mathrm{~cm}$ for a $12 \mathrm{MV}$ photon beam.

\section{Conclusion}

Monte Carlo simulation is an extremely powerful tool in modern radiotherapy. The MCNPX Monte Carlo code offers a rich palette of variance reduction techniques. In this work, several successful variance reduction strategies have been outlined with the aim of reducing CPU time. We found that analogue simulation where both phantom and accelerator head are modeled simultaneously for the use in photon beam dosimetry is not the best method if it is assessed relatively to its computational efficiency. The efficiency of the simulation is increased by a factor greater than 700 when we use the PS method and variance reduction techniques together and the CPU time is considerably reduced. In this work we prove that if variance reduction techniques and PS method are used properly the Monte Carlo calculation efficiency is enhanced without altering the physics of the problem.

Finally, we have studied just a few techniques of variance reduction as BBREM, energy cut-off and PHYS but there are other techniques such as FCL and DXTRAN that we can define as prospects for future paper.

\section{REFERENCES}

[1] B. Habib, B. Poumarede, F. Tola and J. Barthe, "Evaluation of PENFAST a Fast Monte Carlo Code for Dose Calculations in Photon and Electron Radiotherapy Treatment Planning," Physica Medica, Vol. 26, No. 1, 2010, pp. 17-25. doi:10.1016/j.ejmp.2009.03.002

[2] D. Sheikh-Bagheri and D. W. O. Rogers, "Sensitivity of Megavoltage Photon Beam Monte Carlo Simulations to Electron Beam and Other Parameters," Medical Physics, Vol. 29, No. 3, 2002, p. 379.

[3] B. Juste, R. Miró, S. Gallardo, A. Santos and G. Verdú, "Considerations of MCNP Monte Carlo Code to Be Used 
as a Radiotherapy Treatment Planning Tool," 27th Annual Conference of the Engineering in Medicine and Biology, Shanghai, 1-4 September 2005, pp. 2828-2831.

[4] I. Kawrakow and M. Fippel, "Investigation of Variance Reduction Techniques for Monte Carlo Photon Dose Calculation Using XVMC," Physics in Medicine and Biology, Vol. 45, No. 8, 2000, pp. 2163-2183. doi:10.1088/0031-9155/45/8/308

[5] A. Mesbahi, M. Fix, M. Allahverdi, E. Grein and H. Garaati, "Monte Carlo Calculation of Varian 2300C/D Linac Photon Beam Characteristics: A Comparison between MCNP4C, GEANT3 and Measurements," Applied Radiation and Isotopes, Vol. 62, No. 3, 2005, pp. 469477. doi:10.1016/j.apradiso.2004.07.008

[6] D. B. Pelowitz, "MCNPX ${ }^{\mathrm{TM}}$ User's Manual Version 2.5.0," 2005 .

[7] L. Blazy, D. Baltes, J. M. Bordy, D. Cutarella, F. Delaunay and J. Gouriou, "Comparison of PENELOPE Monte Carlo Dose Calculations with Fricke Dosimeter and Ionization Chamber Measurements in Inhomogeneous Phantoms (18 MeV Electron and $12 \mathrm{MV}$ Photon Beams)," Physics in Medicine and Biology, Vol. 51, No. 22, 2006, pp. 5951-5965. doi:10.1088/0031-9155/51/22/016

[8] K. Jabbari, A. Sarfehnia, E. Podgorsak and J. P. Seuntjens, "Monte Carlo Feasibility Study of Orthogonal Bremsstrahlung Beams for Improved Radiation Therapy Imaging," Physics in Medicine and Biology, Vol. 52, No. 4, 2007, p. 1171. doi: $10.1088 / 0031-9155 / 52 / 4 / 021$
[9] A. Ma, J. Awotwi-Pratt, A. Alghamdi, A. Alfuraih and N. M. Spyrou, "Monte Carlo Study of Photoneutron Production in the Varian Clinac 2100C Linac," Radioanalytical and Nuclear Chemistry, Vol. 276, No. 1, 2008, pp. 119123.

[10] S. G. Pareja, M. Vilches and A. M. Lallena, "Ant Colony Method to Control Variance Reduction Techniques in the Monte Carlo Simulation of Clinical Electron Linear Accelerators," Nuclear Instruments and Methods in Physics Research A, Vol. 580, No. 1, 2007, pp. 510-513. doi:10.1016/j.nima.2007.05.217

[11] D. W. O. Rogers, B. A. Faddegon, G. X. Ding, C. M. Ma, J. Wie and T. R. Mackie, "BEAM: A Monte Carlo Code to Simulate Radiotherapy Treatment Units," Medical Physics, Vol. 22, No. 5, 1995, pp. 503-524. doi: $10.1118 / 1.597552$

[12] N. Reynaert, S. van der Marck and D. Schaart, "Monte Carlo Treatment Planning-An Introduction, Nederlandse Commissie Voor Stralingsdosimetrie," Report 16 of the Netherlands Commission on Radiation Dosimetry Netherlands, Commission on Radiation Dosimetry Subcommission Monte Carlo Treatment Planning, 2006.

[13] X-5 Monte Carlo Team MCNP, "A General Monte Carlo N-Particle Transport Code,” Version 5, 2003.

[14] J. K. Shultis and R. E. Faw, “An MCNP Primer,” Kansas State University, Manhattan, 2011. 